\title{
AN ANALYTIC SOLUTION FOR THE TORQUE BETWEEN TWO MAGNETIC DIPOLES
}

\author{
PETER B. LANDECKER*, DANIEL D. VILLANI \\ and KAR W. YUNG \\ Hughes Space and Communications Company, P.O. Box 92919, \\ Los Angeles, CA 90009, USA
}

(Received 21 December 1998; Accepted 1 February 1999)

\begin{abstract}
An analytic equation describing the torque between two magnetic dipoles is derived in this paper. We assume that the dipole sizes are small compared to their separation. Based on a previously derived force calculation between two magnetic dipoles, we derive an analytic expression for the torque. Note that the derived torque decreases with the third power of the distance between the dipoles. It is also interesting to note that the torque on the first dipole is not necessarily equal in magnitude and opposite in direction to the torque on the second dipole. This is due to the torque of both dipoles about a common center of mass.
\end{abstract}

Keywords: Magnetic dipole; Classical electrodynamics; Magnetic force; Magnetic torque

\section{INTRODUCTION}

It is extremely difficult to develop any physical intuition regarding the direction or magnitude of the torques between two magnetic dipoles, except in the cases where the dipole moments are either parallel or perpendicular to the separation vector between the dipoles. A closedform analytic expression would make it much easier to develop this intuition.

* Corresponding author. 
Torques on magnetic dipoles have been previously calculated by Vaidman [1]. However, the torque of one magnetic dipole on another has not yet been derived in electromagnetism textbooks or the periodical literature.

Such an analytic expression of this torque is derived and presented in this paper, for the case of the dipole separation large compared with their sizes.

\section{PREVIOUS WORK ON THE FORCE BETWEEN TWO MAGNETIC DIPOLES}

The magnetic force exerted by a circuit a carrying a current $I_{\mathrm{a}}$ on a circuit b carrying a current $I_{\mathrm{b}}$ (in MKS units) is [2]

$$
\vec{F}_{\mathrm{ab}}=\frac{\mu_{0}}{4 \pi} I_{\mathrm{a}} I_{\mathrm{b}} \oint_{\mathrm{a}} \oint_{\mathrm{b}} \frac{\vec{d}_{\mathrm{b}} \times\left(\vec{d}_{\mathrm{a}} \times \vec{R}\right)}{R^{3}},
$$

where $\mu_{0}$ is the magnetic constant, the permeability of free space, equal to $4 \pi \times 10^{-7} \mathrm{~N} \mathrm{~A}^{-2}, I_{\mathrm{b}}$ is the current of circuit $\mathbf{b}, \vec{d}_{\mathrm{a}}$ is the path increment vector along circuit $\mathbf{a}, \vec{d}_{\mathrm{b}}$ is the path increment vector along circuit $\mathbf{b}, \vec{R}$ is the vector from circuit path element $\vec{d}_{\mathrm{a}}$ to circuit path element $\vec{d}_{\mathrm{b}}$ and $\vec{r}$ is the vector from the center of circuit path element $\vec{d}_{\mathrm{a}}$ to the center of circuit path element $\vec{d}_{\mathrm{b}}$. This geometry is illustrated in Fig. 1.

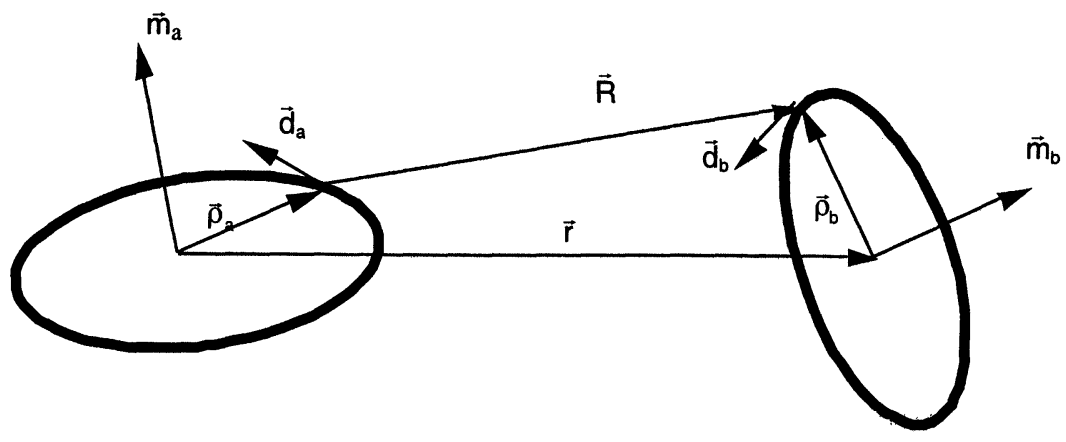

FIGURE 1 Geometry of the two magnetic dipoles. 
The magnetic dipole moments of these two circuits are defined as:

$$
\vec{m}_{\mathrm{a}}=\frac{I_{\mathrm{a}}}{2} \oint \vec{\rho}_{\mathrm{a}} \times \vec{d}_{\mathrm{a}}
$$

and

$$
\vec{m}_{\mathrm{b}}=\frac{I_{\mathrm{b}}}{2} \oint \vec{\rho}_{\mathrm{b}} \times \vec{d}_{\mathrm{b}}
$$

where $\vec{\rho}_{\mathrm{a}}$ is the vector from the center of the magnetic dipole a to circuit element $\vec{d}_{\mathrm{a}}$ and $\vec{\rho}_{\mathrm{b}}$ is the vector from the center of the magnetic dipole $\mathbf{b}$ to circuit element $\vec{d}_{\mathrm{b}}$.

Based on this nomenclature, we derived the final force equation of dipole $\mathbf{a}$ on dipole $\mathbf{b}$ as [2]:

$$
\begin{aligned}
\vec{F}_{\mathrm{ab}}= & \frac{3 \mu_{0} m_{\mathrm{a}} m_{\mathrm{b}}}{4 \pi r^{4}}\left(\hat{r}\left(\hat{m}_{\mathrm{a}} \cdot \hat{m}_{\mathrm{b}}\right)+\hat{m}_{\mathrm{a}}\left(\hat{r} \cdot \hat{m}_{\mathrm{b}}\right)+\hat{m}_{\mathrm{b}}\left(\hat{r} \cdot \hat{m}_{\mathrm{a}}\right)\right. \\
& \left.-5 \hat{r}\left(\hat{r} \cdot \hat{m}_{\mathrm{a}}\right)\left(\hat{r} \cdot \hat{m}_{\mathrm{b}}\right)\right) \\
= & \frac{3 \mu_{0}}{4 \pi r^{4}}\left(\left(\hat{r} \times \vec{m}_{\mathrm{a}}\right) \times \vec{m}_{\mathrm{b}}+\left(\hat{r} \times \vec{m}_{\mathrm{b}}\right) \times \vec{m}_{\mathrm{a}}-2 \hat{r}\left(\vec{m}_{\mathrm{a}} \cdot \vec{m}_{\mathrm{b}}\right)\right. \\
& \left.+5 \hat{r}\left(\hat{r} \times \vec{m}_{\mathrm{a}}\right) \cdot\left(\hat{r} \times \vec{m}_{\mathrm{b}}\right)\right),
\end{aligned}
$$

where the circumflex ${ }^{\wedge}$ indicates a unit vector, and a plain character indicates the magnitude of a vector, e.g. $\vec{r}=r \hat{r}$.

\section{TORQUE BETWEEN TWO MAGNETIC DIPOLES}

To derive the torque, we will find the magnetic field $\vec{B}_{\mathrm{ab}}$ generated by dipole $\mathbf{a}$ at the location of dipole $\mathbf{b}$, and take the cross product of the magnetic moment of dipole $\mathbf{b}$ with the magnetic field created by dipole $\mathbf{a}$. We make use of previously derived [2] relationships for $\vec{\nabla}\left(1 / r^{n}\right)=$ $-n \vec{r} / r^{n+2}$ and $\vec{\nabla}(\vec{m} \cdot \vec{r})=\vec{m}$.

At the location of dipole $\mathbf{b}$, the magnetic field generated by dipole $\mathbf{a}$ is given by [3]:

$$
\begin{aligned}
\vec{B}_{\mathrm{ab}} & =-\frac{\mu_{0}}{4 \pi} \vec{\nabla}\left(\frac{\vec{m}_{\mathrm{a}} \cdot \vec{r}}{r^{3}}\right), \\
& =-\frac{\mu_{0}}{4 \pi}\left[\left(\vec{m}_{\mathrm{a}} \cdot \vec{r}\right) \vec{\nabla}\left(\frac{1}{r^{3}}\right)+\frac{1}{r^{3}} \vec{\nabla}\left(\vec{m}_{\mathrm{a}} \cdot \vec{r}\right)\right],
\end{aligned}
$$




$$
\begin{aligned}
& =-\frac{\mu_{0}}{4 \pi}\left[\left(\vec{m}_{\mathrm{a}} \cdot \vec{r}\right)\left(\frac{-3 \vec{r}}{r^{5}}+\frac{\vec{m}_{\mathrm{a}}}{r^{3}}\right)\right], \\
& =\frac{\mu_{0}}{4 \pi r^{5}}\left[3\left(\vec{m}_{\mathrm{a}} \cdot \vec{r}\right) \vec{r}-r^{2} \vec{m}_{\mathrm{a}}\right] .
\end{aligned}
$$

The resulting torque generated by dipole $\mathbf{a}$ on dipole $\mathbf{b}$ is then given by:

$$
\begin{aligned}
\vec{\tau}_{\mathrm{ab}} & =\vec{m}_{\mathrm{b}} \times \vec{B}_{\mathrm{ab}}, \\
& =\frac{\mu_{0}}{4 \pi r^{5}}\left[3 \vec{m}_{\mathrm{b}} \times\left(\vec{m}_{\mathrm{a}} \cdot \vec{r}\right) \vec{r}-r^{2}\left(\vec{m}_{\mathrm{b}} \times \vec{m}_{\mathrm{a}}\right)\right], \\
& =\frac{\mu_{0} m_{\mathrm{a}} m_{\mathrm{b}}}{4 \pi r^{3}}\left[3\left(\hat{m}_{\mathrm{a}} \cdot \hat{r}\right)\left(\hat{m}_{\mathrm{b}} \times \hat{r}\right)+\left(\hat{m}_{\mathrm{a}} \times \hat{m}_{\mathrm{b}}\right)\right] .
\end{aligned}
$$

Note the asymmetry: if we interchange $\mathbf{a}$ and $\mathbf{b}$ we find that in general, $\left[\vec{\tau}_{\mathrm{ab}} \neq-\vec{\tau}_{\mathrm{ba}}\right]$. It might appear from this asymmetry that the equations violate conservation of angular momentum. However, the total torque on the system (which must sum to zero) includes not only the torques calculated by Eq. (6), which are torques on each dipole about its center of mass, but also a torque on the two-dipole system because the force between the dipoles (Eq. (4)) is not parallel to the vector from the center of one dipole to the other. The cross product $\vec{F}_{\mathrm{ab}} \times \vec{r}$ is in general nonzero. Thus conservation of angular momentum is conserved, after taking into account the angular momentum of both dipoles about their common center of mass. Another way to look at this is to note that $\vec{r} \times \vec{F}_{\mathrm{ab}}+\vec{\tau}_{\mathrm{ab}}+\vec{\tau}_{\mathrm{ba}}=0$, which implies that the net torque on the system equals zero, equivalent to the conservation of total angular momentum.

For various arbitrary combinations of magnetic dipole orientations, both forces and torques can be produced. In a previous companion paper [2], forces between two magnetic dipoles are discussed. The equations in the present paper allow us to understand the torques in fairly simple and intuitive terms. Here, the total torque is proportional to the product of the two magnetic moments, and inversely proportional to the third power of the distance between them. There is (1) a component parallel to the cross product of the two magnetic moments, and (2) a component felt by each individual dipole parallel to the cross product of its dipole moment with the separation vector between the two dipoles. The relative magnitudes of these components is a function of the geometrical relationships between the two dipole moments and between each dipole and the separation vector between them. The asymmetry of the two torques $\tau_{\mathrm{ab}}$ and $\tau_{\mathrm{ba}}$ is due to component (2). 
In addition, we performed typical magnetic torque calculations, using an Excel spreadsheet. Consider the case of two dipoles separated by $1 \mathrm{~m}$ (large compared with the magnetic dipole loop diameters) in $Y$ using an $X Y Z$ coordinate system. Consider also that each dipole has a magnetic moment equal to $100 \mathrm{~A} \mathrm{~m}^{2}$ in magnitude, with the magnetic moment a parallel to $Y$ and the magnetic moment $\mathbf{b}$ parallel to $X$. We then find that both dipoles experience a torque parallel to the $Z$ axis with dipole a exerting a torque of $0.0020 \mathrm{Nm}$ on $\mathbf{b}$, and dipole $\mathbf{b}$ exerting a torque of $0.0010 \mathrm{Nm}$ on $\mathbf{a}$. As has been previously noted, not only do the torques on the dipoles fail to cancel each other, but in this case they are in the same direction and add. However, the total torque on the system is zero because of the sum of the $\vec{r} \times \vec{F}$ torques exerted by each dipole on the other.

\section{CONCLUSIONS}

We derived the analytical expression of the torque between two magnetic dipoles. This torque decreases by the third power of the distance between the two dipoles. Note also the asymmetry in the torque vectors: in general $\left[\vec{\tau}_{\mathrm{ab}} \neq-\vec{\tau}_{\mathrm{ba}}\right]$.

\section{References}

[1] Lev Vaidman, Torque and force on a magnetic dipole, Am. J. Phys. 58 (1990) 978-983.

[2] Kar W. Yung, Peter B. Landecker and Daniel D. Villani, An analytic solution for the force between two magnetic dipoles, J. Mag. Electr. Sep. 9 (1998) 39-52.

[3] Tralli Nunzio, Classical Electromagnetic Theory, New York, McGraw-Hill, 1963, p. 143 , Eqn. (8-5). 